\title{
Ergonomics in Sewing Room
}

\section{Colovic $\mathbf{G}^{*}$}

The College of Textile - Design, Technology and Management, Belgrade, Serbia

*Corresponding author: Colovic G, The College of Textile - Design, Technology and Management, Belgrade, Serbia, Tel: +381 62 8020050; E-mail: gordanacolovic@gmail.com

Rec date: Jul 11, 2014; Acc date: Jul 17, 2014; Pub date: Jul 27, 2014

Copyright: () 2014 Colovic G. This is an open-access article distributed under the terms of the Creative Commons Attribution License, which permits unrestricted use, distribution, and reproduction in any medium, provided the original author and source are credited.

The crucial characteristics of the present time is a rapid technological development, globalization of markets, enterprises that operate without regional and national boundaries, the diversity of views that must also be considered in making decisions, the individualization of business partners and the uncertainty and unpredictability of development, resulting in recent years in almost all areas in the changes that were not there before. New technologies and production processes are rapidly changing the familiar forms of work. Therefore, the work environment must be increasingly adapted to a man in order to perform a work task successfully without excessive fatigue [1].

Proper ergonomic design of each workplace, along with finding suitable methods of work with the appropriate time standards ensures better structure of technological operations with the increased efficiency of sewing machines. Working posture at sewing machines should allow the mobility of the limbs, ergonomically favorable arrangement of working and visible zones and a stable balanced state when performing the work process. Technological processes of sewing clothes are performed on production lines with a large number of technological operations where each technological operation does not last long and has a significant psychological, physical workload for each worker.

The material which goes through the process of work, due to its physical-mechanical characteristics, requires a careful handling when taking, assembling, positioning and putting it aside. Therefore, the structure of technological operations is mostly (65\%) related to the handling of material within support-hand technological grips. The very processing on a machine (sewing grip) is performed during the machine or machine-hand time $(25 \%)$, while $10 \%$ of time is used for non-production work [2].

In designing a workplace it is necessary to pay attention to the conditions of work and harmonize with four characteristics of workers. These are motor-physical (height, weight), sensory (hearing, vision), mental (intellectual ability, memory, attention) and spiritual (morality) characteristics. It is necessary to avoid unnatural body postures, such as leaning on the back or aside, and to lean forward $15^{\circ}$ maximum; working with arms held out, because it reduces the accuracy of work; squatting and stooping. It is necessary to take into consideration the relationship between static and dynamic muscle work (the relationship between the angles of different parts of the body, the mass distribution of individual body segments, the duration of a movement and the risk of a posture) using indirect methods (taking photographs or recording workers), direct methods (watching the man working), subjective methods (analysis of employees, i.e. when a worker is asked about his movements at work).
When designing workplace in the process of sewing it is necessary to achieve dimensional harmony of human-machine system of interphase transport, with the correct physiological posture of sitting, which allows rapid and accurate movements of the motor when switching on the machine and processing the work object, a high level of coordination of movements, a correct position of the spine and good position of the head. The posture of the body of workers, the complexity of the structure of individual movements within the performance of technological operation of sewing and the level of muscular and visual control of the worker depend on the type of technological operation, the type of sewing machine, its technical equipment, machinery and the layout system of workplaces [1].

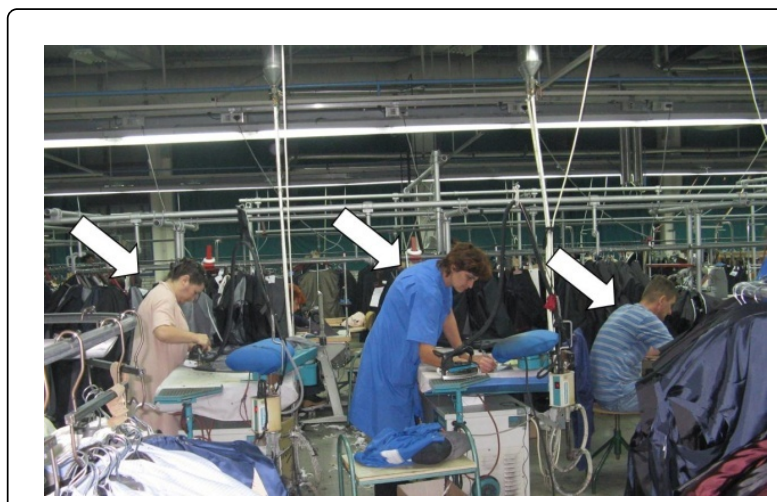

Figure 1: Unnatural body postures

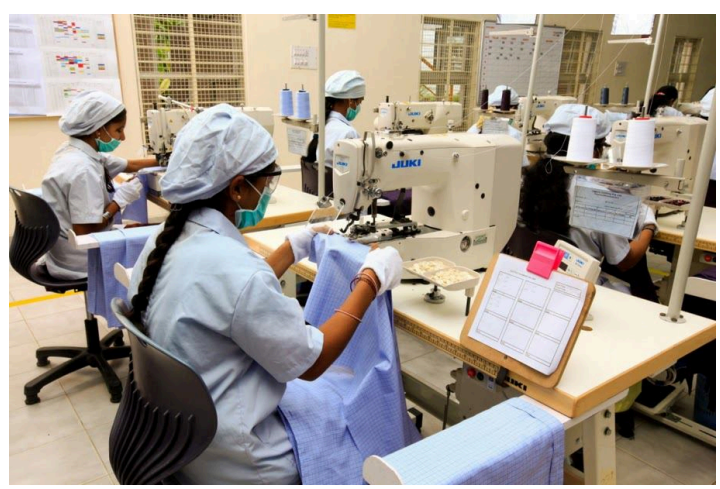

Figure 2: Proper ergonomic workplace 
Page 2 of 2

According to Anderson and Gaardboe, being a sewing machine operator for more than 8 years had a cumulative, permanent and deleterious effect. Poor ergonomic conditions in combination with adverse psychological and social circumstances can cause various diseases, such as: emotional symptoms (psychological tension, uncertainty, anxiety and depression) and psychosomatic diseases (respiratory, gastrointestinal, cardiovascular, the loss or the increase in appetite and sleep) [3].

Sewing involves repeated movements of the shoulder and arm, a static position of the spine and lower extremities, as well as the inclination forward while the job is done throughout the working hours. Sewing machine operators have shoulder and neck problems. Up to $30 \%$ of operators in some garment plants report symptoms of musculoskeletal disorders, such as tingling in the hands and fingers and aches and pains in the arms, shoulders, and neck. Mahone noted that $70 \%$ of sewing machine operators using foot controls report back pain, 35\% report persistent low back pain, $25 \%$ have suffered a compensable cumulative trauma disorder and $49 \%$ of workers experience pain in the neck [4]. So hand sewing and trimming are stressful to all upper limbs, stitching tasks are associated with pain in the shoulders, wrist and hands, ironing by hand is associated with elbow pain, garment assembly tasks are associated with cumulative trauma disorder of hands and wrists and foot operated sewing associated with pain in the back.

Rational usage of working capacity of a man is not only a matter of being humane, but also of being economical. The value of ergonomics goes beyond health and safety. A good ergonomics strategy can add value to a company's business strategy and ultimately contribute to the business goals of higher profits.

\section{References}

1. Colovic G (2014) Ergonomics in the garment industry, Woodhead Publishing Ltd.

2. Colovic G (2010) Management of technology systems in the garment industry, Woodhead Publishing Ltd.

3. Anderson JH, Gaardboe O (1993) Musculoskelatal Disorders of the Neck and upper Limb among Sewing Machine Operators: A Clinical Investigation. Am J Ind Med 24: 689-700.

4. Mahone D (1997) Ergonomics in the Textile and Apparel Industries. CNA Insurance Companies, Chicago. 